\title{
Consumer Acceptance of App Push Notifications: Systematic Review on the Influence of Frequency
}

\author{
https://doi.org/10.3991/ijim.v14i13.14563 \\ Atilla Wohllebe \\ University of Kaposvár, Kaposvár, Hungary \\ atilla.wohllebe@gmail.com
}

\begin{abstract}
Using push notifications, companies can address their smartphone app users with advertising messages. Each of these notifications can mean added value for smartphone users in terms of content, but also disruption and interruption. It is therefore important for advertisers to understand how the frequency of message delivery influences consumer acceptance. The systematic review is based on the PRISMA method and initially identifies a total of 18,725 potentially relevant scientific papers. The 17 research articles included in the qualitative synthesis show that push notifications are suitable for encouraging users to use an app and establish new habits. App usage increases with frequency, with particularly active users tolerating higher frequencies. At the same time it is also shown that too high a frequency can be perceived as disturbing and users should therefore be given the opportunity to determine the frequency with which they receive notifications. A gap in research has been identified in particular in studies that look at real observed behaviour of app users instead of behaviour reported via questionnaires and examine the interaction of frequency, content and presentation of notifications.
\end{abstract}

Keywords - Smartphone Apps, Push Notifications, Consumer Acceptance, Frequency.

\section{Introduction}

With the spread of smartphones worldwide in recent years, they also dominate today's Internet use: In Europe, around 90 percent of the population has access to the Internet [1]. At the same time, Internet use via smartphones now accounts for more than half of the total global traffic volume [2]. Smartphone apps play an important role on the end consumer side: The massive growth in the app market is also raising hopes among many companies that they can profit from this development [3], [4].

A smartphone app is an application for mobile phones that is published by an app developer, often a company, on an app marketplace such as the Apple App Store or the Google Play Store, where users can download and use it on their own mobile phone.

Smartphone apps allow the programmers of the app to send notifications to their users. These notifications are displayed on the lock screen or in the notification bar of the smartphone and are often called "push notifications". Terms such as "mobile push 
notifications" or "push messages" also exist. Based on the S-O-R model, they can be regarded as triggers that influence the consumer as an organism and provoke a reaction [5]. As advertising messages, the notifications can potentially influence the (buying) behaviour of customers [6].

In fact, however, advertising messages or notifications of software applications do not only seem to offer advantages: It has already been proven several times that such forms of user address can also be perceived as annoying and annoying [7] - [10]. In this respect, every benefit from sending a push notification is always accompanied by costs in the sense of a negative user experience [8].

For marketing practitioners, it is therefore highly relevant to weigh up the benefits of advertising effects against the costs of disruptions to customers. This advertising pressure can be defined as frequency or the amount of messages sent per user in a given period of time.

This paper therefore examines the influence of frequency on consumer and user acceptance of push notifications in the context of a systematic review.

\section{Methodology}

For the systematic review the authors apply the PRISMA scheme [11]. The publications included in this work were taken from the scientific database ScienceDirect of the Elsevier publishing house on 1 March 2020.

The following search terms were used to identify potentially relevant papers: "app notifications frequency", "push notifications frequency", "app marketing frequency" and "mobile marketing frequency". "App notifications" and "push notifications" are used virtually synonymously and refer to the messages sent from smartphone apps to the lock screen or notification bar of a smartphone. The term "app marketing", on the other hand, is somewhat broader and can potentially also refer to the marketing of smartphone apps, for example, i.e. the acquisition of new users through app downloads. The term "mobile marketing" is even broader and can potentially also refer to SMS or the control of classic advertising banners on smartphones.

Table 1. Review protocol

\begin{tabular}{|c|c|}
\hline Review question & "How does frequency affect consumer acceptance of push notifications?" \\
\hline \multirow[t]{2}{*}{ Literature search } & Sources: ScienceDirect \\
\hline & $\begin{array}{l}\text { Search phrases: "app notifications frequency", "push notifications frequency", } \\
\text { "app marketing frequency", "mobile marketing frequency" }\end{array}$ \\
\hline Filter criteria & $\begin{array}{l}\text { Type of work: Research articles Years: } 2017-2020 \\
\text { Publication type: open access only }\end{array}$ \\
\hline \multirow[t]{2}{*}{ Exclusions } & $\begin{array}{l}\text { By title: Examination of a thematic reference in broader sense; exclusion of e.g. } \\
\text { technological concepts or articles with exclusively medical reference }\end{array}$ \\
\hline & $\begin{array}{l}\text { By abstract: Exclusion of articles not related to the effect of push notifications in } \\
\text { general or advertising in a broader sense }\end{array}$ \\
\hline Evaluation & $\begin{array}{l}\text { Full-text assessment: Inclusion of only those articles with specific references to the } \\
\text { frequency of advertising messages and push notifications in particular }\end{array}$ \\
\hline
\end{tabular}


Since the research field of push notifications is still relatively new and consumer habits and preferences can change again and again, the potentially relevant work on the filter function will be limited to the years 2017 to 2020 . Furthermore, only research papers marked as "research articles" in the database will be included in the systematic review. Since the results of this systematic review should also be comprehensible to people without paid access to scientific databases, only papers published via open access will be included. Table 1 shows the protocol of the review including filter criteria and exclusions. Table 2 shows the results of the literature search based on the search terms on ScienceDirect.com.

Table 2. Result of the initial literature search on ScienceDirect.com

\begin{tabular}{|l|c|c|c|c|c|}
\hline \multicolumn{1}{|c|}{ Search term } & Total results & Years 2017-2020 & Research articles & Open access & $\begin{array}{c}\text { Results after } \\
\text { filtering }\end{array}$ \\
\hline App notifications frequency & 1,794 & 1,054 & 1,104 & 204 & 112 \\
\hline Push notifications frequency & 2,657 & 811 & 1,418 & 169 & 70 \\
\hline App marketing frequency & 3,058 & 1,636 & 1,942 & 281 & 146 \\
\hline Mobile marketing frequency & 11,198 & 3,555 & 674 & 976 & 344 \\
\hline
\end{tabular}

After removal of the duplicates, 528 of 672 entries remain in the literature database, which are then checked on the basis of title and abstract to see whether a reference to the research question of this systematic review can be established.

After checking the titles, a total of 368 of 528 research articles are removed. 160 papers remain in the literature database.

Among others, the following papers will be removed due to a missing reference in the title to the research question of this article:

- "5G network slicing using SDN and NFV: A survey of taxonomy, architectures and future challenges" [12]

- "Agricultural development addresses food loss and waste while reducing greenhouse gas emissions" [13]

- "Environmental Noise Performance of Rubberized Asphalt Mixtures: Lamia's case study" [14]

- "Public attitudes towards alcohol control policies in Scotland and England: Results from a mixed-methods study" [15]

In particular, on the basis of their title, articles from other scientific fields, such as medicine or biology, for example, or those that explore and discuss technological concepts without producible reference to consumer research in the broadest sense are removed. 


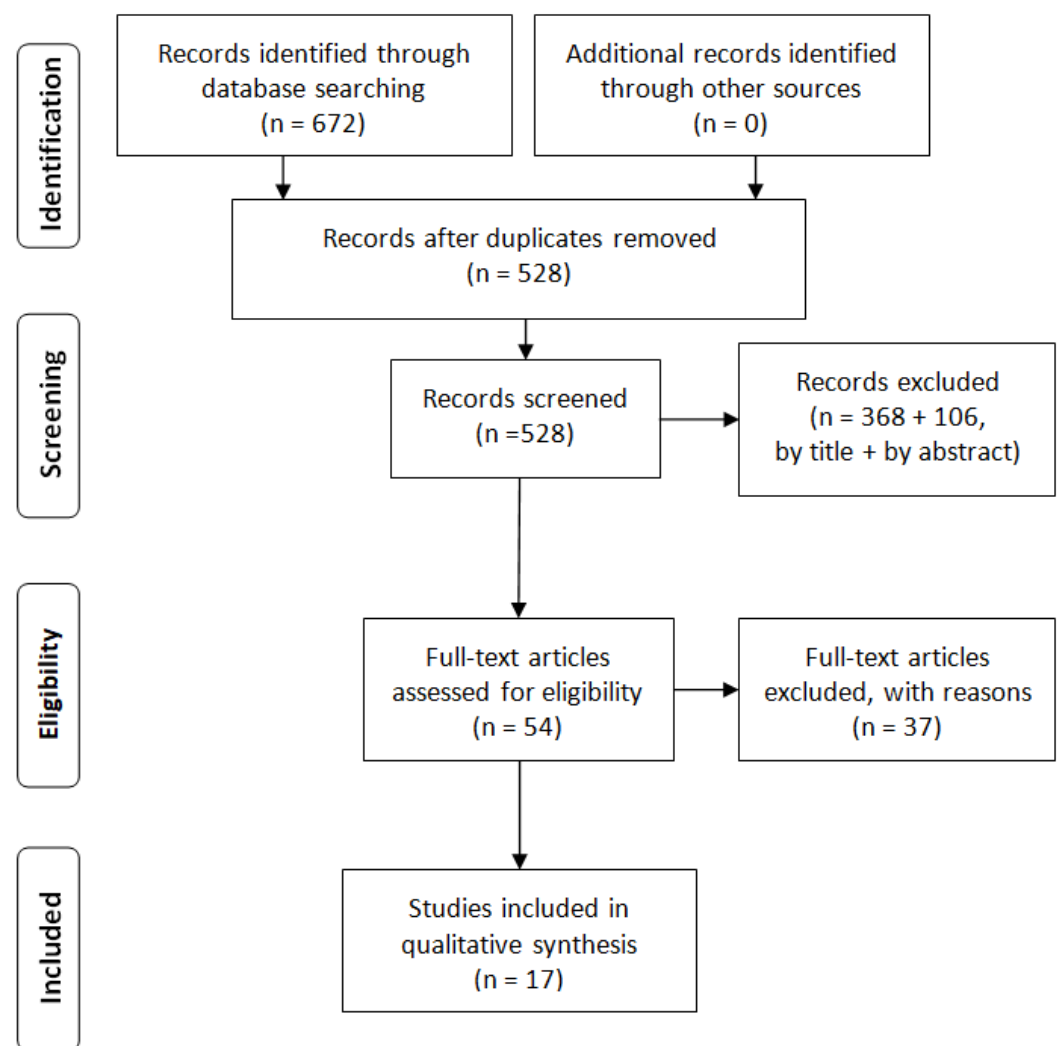

Fig. 1. PRISMA statement (based on [11])

In a subsequent review of the abstracts, 106 of the 160 remaining articles in the literature database are removed in the screening process, so that 54 move on to the next stage, the full-text assessment for eligibility. In particular, articles are excluded here that could have been assumed to have a thematic reference to the research question on the basis of their title, but which then often turned out in the abstract to contain no findings on the effect of the frequency of push notifications or advertising in the broader sense. Examples of this are, for example:

- “Adaptive look-alike targeting in social networks advertising” [16]

- "An affordance perspective of enterprise social media and organizational socialization" [17]

- "An icon that everyone wants to click: How perceived aesthetic qualities predict app icon successfulness" [18]

- "An intelligent approach to design of E-Commerce metasearch and ranking system using next-generation big data analytics" [19] 
A total of 54 full-text articles are assessed for eligibility in the following step. A total of 17 articles, which receive scientific findings linked to the research question of this paper, are tabulated with aim, outcomes, methods, context and, where appropriate, a link to the issue raised here, and included in the qualitative synthesis. The remaining 37 articles are discarded. Figure 1 shows the process of the systematic review according to the PRISMA statement.

\section{Results}

In the following, the previously identified 17 articles are presented in a qualitative synthesis. The articles are presented in the order of the years in which they were published and, in the case of the same year of publication, in alphabetical order of the first named author.

Malik et al. investigate in their article the connection between version updates and downloads of mobile smartphone apps and self-monitoring with a special focus on asthma apps. In an investigation of 5,000 reviews of the top 10 asthma apps in the Google Play Store for Android, they find out in the course of their exploratory work that functions, ratings and downloads are strongly correlated. Also, higher ranked apps show similarities in the especially highlighted features. With regard to the frequency of push notifications, they find that the top-ranked asthma apps have in common that they remind users to take their medication with regular reminders, which is probably one of the most popular functions in this context [20].

Smith et al. are also active in the field of mobile health, who in a case study have demonstrated, documented and tested a development process for a mobile app for behavioral studies. They show how mobile apps can be developed to collect scientific data. As an important function in this context, they identify the regular sending of push notifications to remind subjects, for example, to enter data or answer questions [21].

In their work, Vagrani et al. try to identify factors that cause users to uninstall an app from their smartphone. Against the background of an increasingly shorter application life cycle due to increased competitive pressure, they survey 159 app users. They found that, in particular, too much data consumption, data security concerns, GPS usage and too much memory usage lead to apps being uninstalled. A bad user interface, on the other hand, does not seem to have any influence on the uninstallation of apps. Too frequent notifications and the resulting interruptions or disturbances of users do not seem to have any relevance for the uninstallation of apps [22].

On the basis of an analysis of 18,000 push notifications and 1,414 app users of a mobile health application, Bidargaddi et al. investigate which content types of push notifications contribute positively to engagement. They find that tailored content is more effective than standardised content. At the same time, they also emphasize that less active app users also react less frequently to push notifications. In the context of the frequency of push notifications, they suggest an increased message frequency to activate users who have used an app little or not at all. In this way the engagement of less active users can be increased [23]. 
With regard to the user experience of patients using mobile health applications, Madeira et al. develop a model of how personalization can be used. The theoretically developed model for personalization is then tested on a few patients. In addition to personalizing the content of push notifications, this model also provides for the possibility of personalizing the frequency of push notifications per user [24].

In a comparative examination of web and app, Morrison et al. investigate the effects of digital health behavior interventions in the context of mobile health applications. Based on an observation of 381 web and 162 app users, they find that easy access to features, the application of gamification elements and the use of push notifications ensure that users consume more content. Their results suggest that with the number of push notifications and thus with the frequency, app usage increases [25].

At a nursing college Alsayed et al. investigate how undergraduate nursing students use their smartphone for educational purposes. Their survey of 135 respondents found that over 90 percent always carry their smartphones with them and check for news every morning as soon as they get up. 77.8 percent continue to use it to document information and 24.4 percent say they always use it in a clinical context. With regard to push notifications, it is particularly interesting to note that 65.9 percent state that they always and immediately respond to notifications and 63 percent of respondents have always activated the notifications, which on the one hand offers great potential for high frequencies, but at the same time increases the risk of interference and interruptions due to too many notifications [26].

Using cluster analysis and qualitative analysis across multiple apps, Chen et al. investigate how digital interventions lead to altered health-related behavior. In total, they can identify four different clusters. In particular, users who use an app particularly frequently are particularly enthusiastic about interventions through push notifications. At the same time, some users who use an app less frequently emphasise that they receive too many notifications and therefore perceive the frequency as too high [27].

Heerde et al. are investigating how the use of an app in retail affects the purchasing behavior of customers. Based on the app usage data and the purchasing behaviour of 629 customers who have installed a retailer's app, they find that a smartphone app generates higher sales, especially for offline buying customers. In this context, the authors also find that clear communication towards app users, for which push notifications should be used, increases their acceptance. With regard to frequency, they suggest, for example, that users should be informed when exactly they will receive push notifications when permission is obtained [28].

In the context of using location-based apps to provide information on cultural heritages, McGookin et al. study 45 participants over 5 days: The participants visit an island in Finland and receive push notifications appropriate to their environment. The authors examine how mobile, location-based smartphone apps should be designed in the context of cultural heritages to provide users with interesting information about their surroundings. They conclude that users find the content provided via push notifications interesting, and depending on the local knowledge of the individual users, more indepth content should be provided. Automatically played content, such as video or audio, is perceived by users as annoying. In addition, users would like to be able to delve deeper into the content themselves if they are interested. With regard to push 
notifications, it should be emphasized that users are quite familiar with this medium to be informed about new content and events. They find that users check the app for new content much less frequently on their own initiative than when they receive a corresponding notification. They also stress that users perceive too frequent messages as a disruptive interruption and that the users surveyed would like to see a more even distribution of the frequency of notifications. In addition, they can show that even those users who perceive the frequency as too high appreciate the transmitted content if it is relevant [29].

In a comparative study of Lebanese and British people in the context of mobile banking applications, Mehdi et al. questioned a total of 901 respondents about the factors that contribute to or hinder the adoption of the applications. They find that behavioral intention is influenced by habit, perceived security and perceived privacy as well as trust. With regard to cultural differences, they find that for Lebanese, performance expectancy is important, whereas for British people, price value is important. The authors apply the Unified Theory of Acceptance and Use of Technology (UTAUT2). Their work also contains an indirect reference to the frequency of push notifications: Thus, the frequent use of an application is the basis for the development of a habit and thus the basis for continuous use, so that a continuous addressing of the app users should be helpful [30].

Meijer et al. want to find out to what extent smoking cessation guidelines are already applied and to what extent text messages (SMS) and summary sheets can support this. Over a period of several months, they will monitor healthcare professionals and additionally support these observations through surveys and interviews in order to test learning success and the application of the guidelines on the basis of the messages received. They find that the news is an appropriate tool to train healthcare professionals in the application of the smoker cessation guidelines. Interestingly, those who do not smoke themselves acquire a lot of new knowledge. In the interviews, the respondents sometimes indicate that the frequency of SMS messages, which are displayed on the smartphone as push notifications, is too high. The authors also suggest continuing to send messages after learning success, but with a lower frequency [31].

Noë et al. explore the extent to which a smartphone addiction can be determined based on app usage behavior. After recording the smartphone usage of 64 participants over a period of eight weeks, they conclude that social apps, especially Snapchat, are used particularly frequently in a smartphone addiction. They emphasize that the regular notifications help to bring users back to the apps again and again and that this high frequency of notifications thus binds users permanently to the corresponding apps [32].

In a comparative study of smartphone use during studies in Korea and Australia, Winskel et al. examine the relationship between smartphone use during studies and academic performance. Their survey of 119 Korean and 270 Australian students between 18 and 26 years of age shows that Korean students use their smartphones significantly more during their studies and sometimes at a problematic level compared to Australian students. Overall, the probability of addiction also increases with smartphone use. With regard to push notifications, they note that high frequency can result in inattention and hyperactivity as a constant interruption of smartphone users [33]. 
In a three-step process of model development with validation via a questionnaire, Hsu \& Tang develop a model with the key factors for the mobile app stickiness. Their literature-based development of the model results in a total of six dimensions of the mobile app stickiness: control, communication, context, responsiveness, mobile selfefficiency and advertising. They emphasize that regular reminders from users have a positive effect on consumer interest and identify push notifications as a key factor for the mobile app stickiness [34].

As part of the evaluation of a multimedia marketing campaign, Kikut et al. try to find out which advertising channels are particularly suitable in terms of cost-effectiveness in order to persuade African American patients to participate in the study. They find that radio advertising and postcards are the most suitable channels. Although they do not examine the channel of push notifications, their elaboration contains the important information that the high frequency of radio spots has ensured a correspondingly high coverage, resulting in a strong cost-effectiveness [35]. These findings can certainly be applied to some extent to push notifications.

In their study of 33 disaster-related mobile apps available free of charge in India, Sukhwani \& Shaw come to the conclusion that such apps, which have a long registration process, performance problems or problems loading or send too many push notifications, are less preferred by users. They point out in their study of download numbers, user reviews and functionality that many apps have a very limited reach [36].

\section{$4 \quad$ Summary and Discussion}

A total of 17 papers were examined in synthesis in this systematic review, most of which are directly related to smartphone apps. A large part of the papers examined come from the field of health [20], [21], [23] - [25], [27], [31], [32], [35]. Several papers on smartphone apps in general [22], [34] and from the field of education [26], [33] were also included in the systematic review.

Most of the scientific papers emphasize the suitability of push notifications to encourage users to use apps continuously [20], [21], [23], [25], [29], [30]. In particular, regular notifications can thus also lead to the establishment of a habit, as several authors stress [30], [32], [34]. The frequency of notifications also increases the overall use of the app [25], [29], [32], whereby particularly active users of a smartphone app are more open to higher notification frequencies [27].

Although users react very quickly to the messages [26] and the notifications are at least not one of the main arguments given by users for uninstalling an app [22], the results of the systematic review also show that (especially the high) frequency with which the notifications are sent is not undisputed: Many studies emphasise that push notifications can be perceived by users as disruption and interruption, so that each message can also mean more frustration for users [26], [29], [36]. There may even be a risk that users become more inattentive to their surroundings or develop hyperactivity due to excessive frequency [33]. 
In this respect, it is important that users are informed about the frequency as soon as they obtain permission for push notifications [28]. A personalisable frequency could also contribute to increasing the user experience [24].

At the same time as this systematic review, as with any scientific work, there are some limitations. By means of search terms, a very strong focus on smartphone apps is placed from the outset, so that findings from related areas - e-mail marketing, online banner advertising, dialogue marketing by mail - are not taken into account. In addition, the focus on the topicality of research work and the restriction to articles published in Open Access also leads to a strong limitation that does not take into account findings from the period before 2017 and only considers work that is also available free of charge to scientists and marketing managers without the corresponding access.

In particular, the studies examined show that, with a few exceptions, quantitative analyses based on actual user and usage data are almost completely lacking. In fact, most of the work presented here is based on surveys of users and not on real observed behaviour. Based on the work presented here, there are still large research gaps. In particular, differences between app categories, message content and the design of push notifications based on real user behaviour should be investigated. This would provide marketing managers with helpful, practical scientific findings for their daily work.

\section{Conclusion}

The aim of this paper was to investigate how the frequency of push notifications affects user acceptance. A systematic review identified 18,725 potentially relevant research articles based on a database search. Following the PRISMA scheme, a total of 17 relevant articles were finally considered in the qualitative synthesis. Apart from a restriction to publicly available sources (open access), only very recent work was considered due to the high dynamics in the smartphone and app sector.

In summary, it can be said that push notifications are a central element for activating and binding app users and that the frequency has an important influence on this. In particular, there is a broad consensus that with the increase in the frequency of push notifications, app usage also increases. At the same time, each notification also means a disruption or interruption for the users. In this respect, marketing managers always walk a fine line between value-added information and user disruption. A clear, ideal frequency for sending push notifications cannot be quantified. In particular, however, the tolerance of users seems to increase with the intensity of their own app usage and the added value of the content.

Nevertheless, this paper also shows that most of the research is based on reported behaviour that was collected from consumers by means of questionnaires. In particular, there are still large research gaps in the investigation of the interplay of frequency, content and presentation of push notifications on consumer acceptance. 


\section{$6 \quad$ References}

[1] Eurostat, "Anteil der Internetnutzer in der Europäischen Union (EU-28) nach Ländern im Jahr 2018," Statista, 2018. https://de.statista.com/statistik/daten/studie/184636/umfrage/ internetreichweite-anteil-der-nutzer-in-europa (accessed Dec. 31, 2019).

[2] StatCounter, "Anteil mobiler Endgeräte an allen Seitenaufrufen nach Regionen weltweit im Jahr 2018,” Statista, 2019. https://de.statista.com/statistik/daten/studie/217457/ umfrage/anteil-mobiler-endgeraete-an-allen-seitenaufrufen-weltweit (accessed Dec. 31, 2019).

[3] BITKOM, “Aus Entwicklersicht: Was sind die vorrangigen Gründe für Ihr Unternehmen in die Entwicklung mobiler Anwendungen zu investieren?," Statista, 2011. https://de.statista.com/statistik/daten/studie/196892/umfrage/beweggruende-von-unternehmen-fuer-dieentwicklung-von-apps/ (accessed Oct. 19, 2019). https://doi.org/10.1007/978-3-663-07976 $-7 \quad 2$

[4] BITKOM, "Umsatz mit mobilen Apps in Deutschland in den Jahren 2008 bis 2018 sowie eine Prognose für 2019 (in Millionen Euro)," Statista, 2019. https://de.statista.com/statistik/daten/studie/173810/umfrage/umsatz-mit-mobilen-apps-in-deutschland-seit-2009 (accessed Oct. 19, 2019).

[5] R. S. Woodworth, Psychology: A study of mental life. New York: Henry Holt and Co, 1921.

[6] S. B. MacKenzie and R. J. Lutz, "An Empirical Examination of the Structural Antecedents of Attitude toward the Ad in an Advertising Pretesting Context," Journal of Marketing, vol. 53, no. 2, p. 48, Apr. 1989, https://doi.org/10.2307/1251413

[7] D. C. McFarlane, "Comparison of Four Primary Methods for Coordinating the Interruption of People in Human-Computer Interaction," Human-Computer Interaction, vol. 17, no. 1, pp. 63-139, Mar. 2002, https://doi.org/10.1207/s15327051hci1701 2

[8] J. E. Fischer, N. Yee, V. Bellotti, N. Good, S. Benford, and C. Greenhalgh, "Effects of content and time of delivery on receptivity to mobile interruptions," in Proceedings of the 12th international conference on Human computer interaction with mobile devices and services - MobileHCI '10, Lisbon, Portugal, 2010, p. 103, https://doi.org/10.1145/1851600.18 $\underline{51620}$

[9] A. Sahami Shirazi, N. Henze, T. Dingler, M. Pielot, D. Weber, and A. Schmidt, "Large-scale assessment of mobile notifications," in Proceedings of the 32nd annual ACM conference on Human factors in computing systems - CHI '14, Toronto, Ontario, Canada, 2014, pp. 30553064, https://doi.org/10.1145/2556288.2557189

[10] S. T. Iqbal and E. Horvitz, "Disruption and recovery of computing tasks: field study, analysis, and directions," in Proceedings of the SIGCHI Conference on Human Factors in Computing Systems - CHI '07, San Jose, California, USA, 2007, pp. 677-686, https://doi. org/10.1145/1240624.1240730

[11] D. Moher, A. Liberati, J. Tetzlaff, D. G. Altman, and T. P. Group, "Preferred Reporting Items for Systematic Reviews and Meta-Analyses: The PRISMA Statement," PLOS Medicine, vol. 6, no. 7, p. e1000097, Jul. 2009, https://doi.org/10.1371/journal.pmed.1000097.

[12] A. A. Barakabitze, A. Ahmad, R. Mijumbi, and A. Hines, "5G network slicing using SDN and NFV: A survey of taxonomy, architectures and future challenges," Computer Networks, vol. 167, p. 106984, 2020, https://doi.org/10.1016/j.comnet.2019.106984

[13] G. L. Galford et al., "Agricultural development addresses food loss and waste while reducing greenhouse gas emissions," Science of The Total Environment, vol. 699, p. 134318, 2020, https://doi.org/10.1016/j.scitotenv.2019.134318 
[14] S. Mavridou and F. Kehagia, "Environmental Noise Performance of Rubberized Asphalt Mixtures: Lamia's case study," Procedia Environmental Sciences, vol. 38, pp. 804-811, 2017, https://doi.org/10.1016/j.proenv.2017.03.165

[15] J. Li et al., "Public attitudes towards alcohol control policies in Scotland and England: Results from a mixed-methods study," Social Science \& Medicine, vol. 177, pp. 177-189, 2017, https://doi.org/10.1016/j.socscimed.2017.01.037

[16] A. Popov and D. Iakovleva, "Adaptive look-alike targeting in social networks advertising," Procedia Computer Science, vol. 136, pp. 255-264, 2018, https://doi.org/10.1016/j.procs. $\underline{2018.08 .264}$

[17] D. E. Leidner, E. Gonzalez, and H. Koch, "An affordance perspective of enterprise social media and organizational socialization," The Journal of Strategic Information Systems, vol. 27, no. 2, pp. 117-138, 2018, https://doi.org/10.1016/j.jsis.2018.03.003

[18] H. Jylhä and J. Hamari, "An icon that everyone wants to click: How perceived aesthetic qualities predict app icon successfulness," International Journal of Human-Computer Studies, vol. 130, pp. 73-85, 2019, https://doi.org/10.1016/j.ijhcs.2019.04.004.

[19] D. Malhotra and O. P. Rishi, "An intelligent approach to design of E-Commerce metasearch and ranking system using next-generation big data analytics," Journal of King Saud University - Computer and Information Sciences, 2018, https://doi.org/10.1016/j. jksuci.2018.02.015

[20] H. Malik, E. M. Shakshuki, and S. Katuku, "Exploring the Relationship Between Version Updates and Downloads of Asthma Mobile Apps," Procedia Computer Science, vol. 109, pp. 624-631, 2017, https://doi.org/10.1016/j.procs.2017.05.368

[21] A. Smith, K. de Salas, I. Lewis, and B. Schüz, "Developing smartphone apps for behavioural studies: The AlcoRisk app case study," Journal of Biomedical Informatics, vol. 72, pp. 108119, 2017, https://doi.org/10.1016/j.jbi.2017.07.007

[22] A. Vagrani, N. Kumar, and P. V. Ilavarasan, "Decline in Mobile Application Life Cycle," Procedia Computer Science, vol. 122, pp. 957-964, 2017, https://doi.org/10.1016/j.procs. $\underline{2017.11 .460}$

[23] N. Bidargaddi, T. Pituch, H. Maaieh, C. Short, and V. Strecher, "Predicting which type of push notification content motivates users to engage in a self-monitoring app," Preventive Medicine Reports, vol. 11, pp. 267-273, 2018, https://doi.org/10.1016/j.pmedr.2018.07. $\underline{004}$

[24] R. N. Madeira, H. Germano, P. Macedo, and N. Correia, "Personalising the User Experience of a Mobile Health Application towards Patient Engagement," Procedia Computer Science, vol. 141, pp. 428-433, 2018, https://doi.org/10.1016/j.procs.2018.10.173

[25] L. G. Morrison et al., "Comparing usage of a web and app stress management intervention: An observational study," Internet Interventions, vol. 12, pp. 74-82, 2018, https://doi.org/ 10.1016/j.invent.2018.03.006

[26] S. Alsayed, N. Bano, and H. Alnajjar, "Evaluating practice of smartphone use among university students in undergraduate nursing education," Health Professions Education, 2019, https://doi.org/10.1016/j.hpe.2019.06.004

[27] A. T. Chen, S. Wu, K. N. Tomasino, E. G. Lattie, and D. C. Mohr, "A multi-faceted approach to characterizing user behavior and experience in a digital mental health intervention," Journal of Biomedical Informatics, vol. 94, p. 103187, 2019, https://doi.org/10. 1016/j.jbi.2019.103187

[28] H. J. van Heerde, I. M. Dinner, and S. A. Neslin, "Engaging the unengaged customer: The value of a retailer mobile app," International Journal of Research in Marketing, vol. 36, no. 3, pp. 420-438, 2019, https://doi.org/10.1016/j.ijresmar.2019.03.003 
[29] D. McGookin, K. Tahiroğlu, T. Vaittinen, M. Kytö, B. Monastero, and J. C. Vasquez, "Investigating tangential access for location-based digital cultural heritage applications," International Journal of Human-Computer Studies, vol. 122, pp. 196-210, 2019, https://doi. org/10.1016/j.ijhcs.2018.09.009.

[30] M. Merhi, K. Hone, and A. Tarhini, "A cross-cultural study of the intention to use mobile banking between Lebanese and British consumers: Extending UTAUT2 with security, privacy and trust," Technology in Society, vol. 59, p. 101151, 2019, https://doi.org/10.1016/ j.techsoc.2019.101151

[31] E. Meijer, N. H. Chavannes, D. Segaar, J. L. Parlevliet, and R. M. J. J. V. D. Kleij, “Optimizing smoking cessation guideline implementation using text-messages and summarysheets: A mixed-method evaluation," Clinical eHealth, vol. 2, pp. 16-24, 2019, https://doi.org/10.1016/j.ceh.2019.08.001

[32] B. Noë, L. D. Turner, D. E. J. Linden, S. M. Allen, B. Winkens, and R. M. Whitaker, "Identifying Indicators of Smartphone Addiction Through User-App Interaction," Computers in Human Behavior, vol. 99, pp. 56-65, 2019, https://doi.org/10.1016/j.chb.2019.04. $\underline{023}$.

[33] H. Winskel, T.-H. Kim, L. Kardash, and I. Belic, "Smartphone use and study behavior: A Korean and Australian comparison," Heliyon, vol. 5, no. 7, p. e02158, 2019, https://doi. org/10.1016/j.heliyon.2019.e02158

[34] T.-H. Hsu and J.-W. Tang, "Development of hierarchical structure and analytical model of key factors for mobile app stickiness," Journal of Innovation \& Knowledge, vol. 5, no. 1, pp. 68-79, 2020, https://doi.org/10.1016/j.jik.2019.01.006.

[35] A. Kikut et al., "Evaluation of a multimedia marketing campaign to engage African American patients in glaucoma screening," Preventive Medicine Reports, vol. 17, p. 101057, 2020, https://doi.org/10.1016/j.pmedr.2020.101057

[36] V. Sukhwani and R. Shaw, "Operationalizing crowdsourcing through mobile applications for disaster management in India," Progress in Disaster Science, vol. 5, p. 100052, 2020, https://doi.org/10.1016/j.pdisas.2019.100052

\section{Author}

Atilla Wohllebe is a PhD student at the Doctoral School in Management and Organizational Sciences, University of Kaposvár, Hungary. Living and working in Hamburg, Germany, he is engaged in research and professional work on consumer interactions with smartphone apps. Atilla Wohllebe holds a B.A. in Business Administration and an M.Sc. in E-Commerce.

Article submitted 2020-04-02. Resubmitted 2020-05-13. Final acceptance 2020-05-23. Final version published as submitted by the authors. 